\title{
Numerical analysis for time-fractional Schrödinger equation on two space dimensions
}

\author{
Jun Zhang ${ }^{1,2}$, JinRong Wang ${ }^{1,3^{*}}$ and Yong Zhou ${ }^{4,5}$
}

\section{"Correspondence:}

jrwang@gzu.edu.cn

'Department of Mathematics, Guizhou University, Guiyang, China

${ }^{3}$ School of Mathematical Sciences, Qufu Normal University, Qufu, China Full list of author information is available at the end of the article

\section{Springer}

\begin{abstract}
In this paper, we study the numerical methods for solving the time-fractional Schrödinger equation (TFSE) with Caputo or Riemann-Liouville fractional derivative. The numerical schemes are implemented by using the $L 1$ scheme in time direction and Fourier-Galerkin/Legendre-Galerkin spectral methods in spatial variable. We prove that the two schemes are unconditionally stable and numerical solutions converge with the order $\mathcal{O}\left(\Delta t^{2-\alpha}+N^{-s}+N^{-m}\right)$, where $\alpha$ is the order of the fractional derivative, $\Delta t, N$ are the step of time and polynomial degree, respectively, $m, s$ are the regularity of $u$ and $V$. Several numerical results are performed to confirm the theoretical analysis.
\end{abstract}

MSC: $65 \mathrm{~N} 15 ; 65 \mathrm{~N} 30$

Keywords: Schrödinger equation; Caputo; Riemann-Liouville; Fourier-spectral method; Error estimate

\section{Introduction}

In this work, we construct numerical methods to solve the following TFSE:

$$
\frac{1}{i} \frac{\partial^{\alpha} u}{\partial t^{\alpha}}-\Delta u+\beta V(x, y) u=0 \quad \text { in } \Omega \times[0, T],
$$

(i) $u$ are periodic; or (ii) $\left.u\right|_{\partial \Omega}=0$,

$$
u(x, y, 0)=u_{0}(x, y) \quad \text { on } \partial \Omega \times[0, T] \text {, }
$$

where $i^{2}=-1$, $\frac{\partial^{\alpha} u}{\partial t^{\alpha}}(0<\alpha<1)$ denotes the Caputo or Riemann-Liouville fractional derivative, $\Omega \subset \mathbf{R}^{2}$ is a bound domain, $\beta$ is a positive constant, and $V$ represents a potential function.

TFSE can be viewed as a generalization of the classical Schrödinger equation. It has emerged as an appropriate model in various applications, such as plasma physics, polymer physics, nonlinear optic, etc. The concept of fractal in quantum mechanics has been developed over the past ten years, since Laskin $[1,2]$ defined some path integrals and developed the space fractional quantum mechanics on the basis of new fractional path

(c) The Author(s) 2020. This article is licensed under a Creative Commons Attribution 4.0 International License, which permits use, sharing, adaptation, distribution and reproduction in any medium or format, as long as you give appropriate credit to the original author(s) and the source, provide a link to the Creative Commons licence, and indicate if changes were made. The images or other third party material in this article are included in the article's Creative Commons licence, unless indicated otherwise in a credit line to the material. If material is not included in the article's Creative Commons licence and your intended use is not permitted by statutory regulation or exceeds the permitted use, you will need to obtain permission directly from the copyright holder. To view a copy of this licence, visit http://creativecommons.org/licenses/by/4.0/ 
integrals method. Naber [3], Wang and Xu [4] constructed a class of TFSE with Caputo fractional derivative and discussed the solutions for a free particle and a potential well. Guo and $\mathrm{Xu}$ [5] studied the TSFE with a free particle, and they obtained the fundamental solution of the problem. Cheng [6] proved the existence of ground state for TSFE with unbounded potential by using Lagrange multiplier method. In [7], Felmer and Tan studied the existence, regularity of the ground state for the nonlinear fractional Schrödinger equation. It is remarkable that Wang et al. [8] investigated the existence and uniqueness of optimal controls of TFSE (1)-(3).

It is difficult to find an explicit form of analytical solutions of fractional equation, so some recent contributions have focused on using numerical methods to obtain approximate solutions. Rida et al. [9] proposed an Adomian decomposition method for solving nonlinear TFSE. Li and Xu [10] constructed a time-space spectral method to investigate the solution of fractional partial differential equations. Yildirim [11] introduced a homotopy perturbation method to study analytical solutions for fractional Schrödinger equation. Wei et al. $[12,13]$ presented a local discontinuous Galerkin (LDG) method to approximate the solution of TFSE. Mohebbi et al. [14] developed a shifted Legendre collocation method to solve TFSE with initial-boundary and nonlocal conditions. Baleanu et al. [15-17] investigated the soliton solutions of the nonlinear Schrödinger equation with Kerr law nonlinearity. They obtained the exact dark optical, dark-singular, and periodic singular soliton solutions of the equation. Garrappa et al. [18] discussed approximating the solution of TFSE by using the Krylov projection methods. Zhu et al. [19] presented a finite element method to solve time-space-fractional Schrödinger equation with Caputo and Riesz derivatives. The other related numerical methods for fractional equation can been found in [20-25] and the references therein.

On the other hand, L1 scheme $[26,27]$ is an efficient numerical method to approximate Caputo or Riemann-Liouville derivative. Langlands and Henry [28], Sun and Wu [29], Lin and $\mathrm{Xu}$ [30] obtained the error estimate of the L1 scheme. Grajales and Vargas [31] constructed a Crank-Nicholson/Fourier-Galerkin method to approximate the solution of the Schrödinger equation. Gong et al. [32] proposed an energy conservative CrankNicholson/Fourier pseudo-spectral method to solve the Schrödinger equation. Kumar et al. [33-36] introduced a series of homotopy transform methods to solve some fractional equations.

As a classical high-order method, spectral method has been widely used to solve PDE/ODE equations. In this article, we propose two efficient numerical schemes to approximate the TFSE with Caputo or Riemann-Liouville derivative. The proposed schemes are performed by combining the $\mathrm{L} 1 \mathrm{scheme}$ for fractional derivative and FourierGalerkin/Legendre-Galerkin spectral methods for space variable. A detailed analysis of the numerical scheme is provided for both stability and error estimate. Our rigorous analysis results show that numerical methods lead to 2- $\alpha$ order accuracy in time direction and spectral accuracy in space direction. At last, some numerical examples are conducted to support the theoretical claims.

The rest of the paper is structured in the following way. Section 2 introduces the L1 scheme for Caputo and Riemann-Liouville derivative. In Sect. 3, we discuss error estimates for the full discrete schemes. In Sect. 4, some numerical experiments are presented to illustrate the validity of the numerical method. The conclusions of this paper are given in Sect. 5. 


\section{Stability for semi-discretization TFSE}

In this part, we present the semi-discrete schemes for the solution of (1)-(3). First, we introduce an L1 scheme to discrete the Caputo and Riemann-Liouville derivatives. Let $M$ be a positive integer, $\Delta t=T / M$ be the time step, and $t_{n}=n \Delta t, n=0,1, \ldots, M-1$ be a mesh point. We introduce the following L1 scheme for Caputo fractional derivative of order $\alpha$ :

$$
\frac{1}{\Gamma(2-\alpha)} \sum_{j=0}^{n} b_{j} \frac{u\left(t_{n+1-j}\right)-u\left(t_{n-j}\right)}{\Delta t^{\alpha}}
$$

and the Riemann-Liouville derivative is approximated by

$$
\frac{1}{\Gamma(2-\alpha)} \sum_{j=0}^{n} b_{j} \frac{u\left(t_{n+1-j}\right)-u\left(t_{n-j}\right)}{\Delta t^{\alpha}}+\frac{u\left(t_{0}\right)\left(t_{n+1}-t_{0}\right)^{-\alpha}}{\Gamma(1-\alpha)},
$$

where $b_{j}=(j+1)^{1-\alpha}-j^{1-\alpha}$.

Then, we rewrite the complex function $u(x, y, t)$ into the real part and the imaginary part, that is, $u(x, y, t)=v(x, y, t)+i w(x, y, t)$. Then we get the following coupled system:

$$
\left\{\begin{array}{l}
\frac{\partial^{\alpha} v}{\partial t^{\alpha}}+\Delta w-\beta V w=0 \\
\frac{\partial^{\alpha} w}{\partial t^{\alpha}}-\Delta v+\beta V v=0
\end{array}\right.
$$

Remark 1 It is worth mentioning that there are two different boundary conditions for Caputo and Riemann-Liouville derivatives.

Then we obtain the following semi-discrete schemes for TFSE with Caputo derivative:

$$
\left\{\begin{array}{l}
\left(v^{n+1}-\sum_{j=0}^{n-1}\left(b_{j}-b_{j+1}\right) v^{n-j}-b_{n} v^{0}\right)+a_{0} \Delta w^{n+1}-a_{0} \beta V w^{n+1}=0 \\
\left(w^{n+1}-\sum_{j=0}^{n-1}\left(b_{j}-b_{j+1}\right) w^{n-j}-b_{n} w^{0}\right)-a_{0} \Delta v^{n+1}+a_{0} \beta V v^{n+1}=0
\end{array}\right.
$$

and Riemann-Liouville derivative:

$$
\left\{\begin{array}{l}
\left(v^{n+1}-\sum_{j=0}^{n-1}\left(b_{j}-b_{j+1}\right) v^{n-j}-\bar{b}_{n} v^{0}\right)+a_{0} \Delta w^{n+1}-a_{0} \beta V w^{n+1}=0, \\
\left(w^{n+1}-\sum_{j=0}^{n-1}\left(b_{j}-b_{j+1}\right) w^{n-j}-\bar{b}_{n} w^{0}\right)-a_{0} \Delta v^{n+1}+a_{0} \beta V v^{n+1}=0
\end{array}\right.
$$

where $a_{0}=\Delta t^{\alpha} \Gamma(2-\alpha), \bar{b}_{n}=b_{n}-(1-\alpha) \Delta t^{\alpha} t_{n+1}^{-\alpha}$.

Lemma 1 For all $\bar{b}_{n}, n \geq 0$, we have

$$
0<\bar{b}_{n} \leq \alpha b_{n} .
$$

Proof It is easy to check that

$$
\bar{b}_{n}=(n+1)^{1-\alpha}-n^{1-\alpha}-(1-\alpha)(n+1)^{-\alpha} .
$$

Set

$$
f(t)=(1+t)^{\alpha}-(1+t \alpha), \quad t \geq 0 .
$$


Then $f(0)=0$ and $f^{\prime}(t)=\alpha\left((1+t)^{\alpha-1}-1\right)<0, \forall t>0$, thus $f(t)<f(0)=0$, then

$$
(1+t)^{\alpha}<(1+t \alpha), \quad \forall t>0
$$

Let $t=\frac{1}{x}, x>0$, we have

$$
\left(1+\frac{1}{x}\right)^{\alpha}<\left(1+\frac{\alpha}{x}\right)
$$

Therefore

$$
\left(\frac{1+x}{x}\right)^{\alpha}<\left(\frac{x+\alpha}{x}\right) .
$$

That is

$$
(1+x)^{-\alpha}(x+\alpha)>x^{1-\alpha}
$$

Hence

$$
(1+x)^{-\alpha}(1+x-(1-\alpha))>x^{1-\alpha} .
$$

Namely

$$
(1+x)^{1-\alpha}-(1-\alpha)(1+x)^{1-\alpha}-x^{1-\alpha}>0 .
$$

On the other hand, there holds

$$
\bar{b}_{n}-\alpha b_{n}=(1-\alpha)\left((n+1)^{1-\alpha}-n^{1-\alpha}-(n+1)^{-\alpha}\right) \leq 0, \quad \forall n \geq 0 .
$$

The above inequalities can be proved as follows:

$$
(n+1)^{1-\alpha}-n^{1-\alpha}-(n+1)^{-\alpha} \leq 0 .
$$

It follows that

$$
(n+1)^{1-\alpha}-(n+1)^{-\alpha} \leq n^{1-\alpha} .
$$

Thus, we arrive at

$$
(n+1)^{1-\alpha}\left(1-\frac{1}{n+1}\right) \leq n^{1-\alpha} .
$$

That is

$$
(n+1)^{-\alpha} \leq n^{-\alpha} .
$$

Hence, we finish the proof of (9).

We have the following unconditional stability results. 
Lemma 2 The semi-discrete schemes (7) are unconditionally stable such that, for $0 \leq n \leq$ $M-1$, we have

$$
\left\|v^{n+1}\right\|^{2}+\left\|w^{n+1}\right\|^{2} \leq\left\|v^{0}\right\|^{2}+\left\|w^{0}\right\|^{2} .
$$

Proof When $n=0$, computing the $L^{2}$ inner product of (7) with $2 v^{1}$ and $2 w^{1}$, we obtain

$$
\begin{aligned}
& \left(v^{1}-v^{0}, 2 v^{1}\right)+2 a_{0}\left(\Delta w^{1}, v^{1}\right)-2 a_{0} \beta\left(V w^{1}, v^{1}\right)=0, \\
& \left(w^{1}-w^{0}, 2 w^{1}\right)-2 a_{0}\left(\Delta v^{1}, w^{1}\right)+2 a_{0} \beta\left(V v^{1}, w^{1}\right)=0 .
\end{aligned}
$$

This yields

$$
\left\|v^{1}\right\|^{2}-\left\|v^{0}\right\|^{2}+\left\|v^{1}-v^{0}\right\|^{2}+\left\|w^{1}\right\|^{2}-\left\|w^{0}\right\|^{2}+\left\|w^{1}-w^{0}\right\|^{2}=0 .
$$

That is

$$
\left\|v^{1}\right\|^{2}+\left\|w^{1}\right\|^{2} \leq\left\|v^{0}\right\|^{2}+\left\|w^{0}\right\|^{2}
$$

Assume that the following inequality holds:

$$
\left\|v^{j}\right\|^{2}+\left\|w^{j}\right\|^{2} \leq\left\|v^{0}\right\|^{2}+\left\|w^{0}\right\|^{2}, \quad j=2,3, \ldots, n
$$

Then we need to prove $\left\|v^{n+1}\right\|^{2}+\left\|w^{n+1}\right\|^{2} \leq\left\|v^{0}\right\|^{2}+\left\|w^{0}\right\|^{2}$. When $j=n+1$, computing the $L^{2}$ inner product of (7) with $2 v^{n+1}$ and $2 w^{n+1}$, we derive

$$
\begin{aligned}
2\left\|v^{n+1}\right\|^{2}+2\left\|w^{n+1}\right\|^{2} & \\
= & 2\left(\sum_{j=0}^{n-1}\left(b_{j}-b_{j+1}\right) v^{n-j}+b_{n} v^{0}, v^{n+1}\right)+2\left(\sum_{j=0}^{n-1}\left(b_{j}-b_{j+1}\right) w^{n-j}+b_{n} w^{0}, w^{n+1}\right) \\
\leq & \left.\sum_{j=0}^{n-1}\left(b_{j}-b_{j+1}\right)\left(\left\|v^{n-j}\right\|^{2}+\left\|v^{n+1}\right\|^{2}\right)+b_{n}\left(\left\|v^{0}\right\|^{2}+\left\|v^{n+1}\right\|^{2}\right)\right) \\
& \left.+\sum_{j=0}^{n-1}\left(b_{j}-b_{j+1}\right)\left(\left\|w^{n-j}\right\|^{2}+\left\|w^{n+1}\right\|^{2}\right)+b_{n}\left(\left\|w^{0}\right\|^{2}+\left\|w^{n+1}\right\|^{2}\right)\right) .
\end{aligned}
$$

Noting that

$$
\sum_{j=0}^{n-1}\left(b_{j}-b_{j+1}\right)+b_{n}=1 \text {. }
$$

Thus, we get

$$
\begin{aligned}
\left\|v^{n+1}\right\|^{2}+\left\|w^{n+1}\right\|^{2} & \leq \sum_{j=0}^{n-1}\left(b_{j}-b_{j+1}\right)\left(\left\|v^{n-j}\right\|^{2}+\left\|w^{n-j}\right\|^{2}\right)+b_{n}\left(\left\|v^{0}\right\|^{2}+\left\|w^{0}\right\|^{2}\right) \\
& \leq\left(\sum_{j=0}^{n-1}\left(b_{j}-b_{j+1}\right)+b_{n}\right)\left(\left\|v^{0}\right\|^{2}+\left\|w^{0}\right\|^{2}\right)
\end{aligned}
$$




$$
=\left\|v^{0}\right\|^{2}+\left\|w^{0}\right\|^{2} .
$$

The proof is completed.

Lemma 3 Semi-discrete equations (8) are unconditionally stable, and $v^{n+1}, w^{n+1}$ satisfy

$$
\left\|v^{n+1}\right\|^{2}+\left\|w^{n+1}\right\|^{2} \leq \alpha\left\|v^{0}\right\|^{2}+\alpha\left\|w^{0}\right\|^{2}, \quad 0 \leq n \leq M-1 .
$$

Proof For $n=0$, taking the $L^{2}$ inner product of (8) with $2 v^{1}, 2 w^{1}$, we get

$$
\begin{aligned}
& \left(v^{1}-\alpha v^{0}, 2 v^{1}\right)+2 a_{0}\left(\Delta w^{1}, v^{1}\right)-2 a_{0} \beta\left(V w^{1}, v^{1}\right)=0, \\
& \left(w^{1}-\alpha w^{0}, 2 w^{1}\right)-2 a_{0}\left(\Delta v^{1}, w^{1}\right)+2 a_{0} \beta\left(V v^{1}, w^{1}\right)=0 .
\end{aligned}
$$

Consequently,

$$
\left\|v^{1}\right\|^{2}-\alpha^{2}\left\|v^{0}\right\|^{2}+\left\|v^{1}-\alpha v^{0}\right\|^{2}+\left\|w^{1}\right\|^{2}-\alpha^{2}\left\|w^{0}\right\|^{2}+\left\|w^{1}-\alpha w^{0}\right\|^{2}=0 .
$$

We arrive at

$$
\left\|v^{1}\right\|^{2}+\left\|w^{1}\right\|^{2} \leq \alpha\left\|v^{0}\right\|^{2}+\alpha\left\|w^{0}\right\|^{2}
$$

Suppose

$$
\left\|v^{j}\right\|^{2}+\left\|w^{j}\right\|^{2} \leq \alpha\left\|v^{0}\right\|^{2}+\alpha\left\|w^{0}\right\|^{2}, \quad j=2,3, \ldots, n .
$$

For $k=n+1$, taking the $L^{2}$ inner product of (7) with $2 v^{n+1}$ and $2 w^{n+1}$, we find that

$$
\begin{aligned}
2\left\|v^{n+1}\right\|^{2}+2\left\|w^{n+1}\right\|^{2} & \\
= & 2\left(\sum_{j=0}^{n-1}\left(b_{j}-b_{j+1}\right) v^{n-j}+\bar{b}_{n} v^{0}, v^{n+1}\right)+2\left(\sum_{j=0}^{n-1}\left(b_{j}-b_{j+1}\right) w^{n-j}+\bar{b}_{n} w^{0}, w^{n+1}\right) \\
\leq & \left.\sum_{j=0}^{n-1}\left(b_{j}-b_{j+1}\right)\left(\left\|v^{n-j}\right\|^{2}+\left\|v^{n+1}\right\|^{2}\right)+\bar{b}_{n}\left(\left\|v^{0}\right\|^{2}+\left\|v^{n+1}\right\|^{2}\right)\right) \\
& \left.+\sum_{j=0}^{n-1}\left(b_{j}-b_{j+1}\right)\left(\left\|w^{n-j}\right\|^{2}+\left\|w^{n+1}\right\|^{2}\right)+\bar{b}_{n}\left(\left\|w^{0}\right\|^{2}+\left\|w^{n+1}\right\|^{2}\right)\right) .
\end{aligned}
$$

By using (9), we have

$$
\begin{aligned}
\left\|v^{n+1}\right\|^{2}+\left\|w^{n+1}\right\|^{2} & \leq \sum_{j=0}^{n-1}\left(b_{j}-b_{j+1}\right)\left(\left\|v^{n-j}\right\|^{2}+\left\|w^{n-j}\right\|^{2}\right)+\bar{b}_{n}\left(\left\|v^{0}\right\|^{2}+\left\|w^{0}\right\|^{2}\right) \\
& \leq\left(\sum_{j=0}^{n-1}\left(b_{j}-b_{j+1}\right)\right)\left(\alpha\left\|v^{0}\right\|^{2}+\alpha\left\|w^{0}\right\|^{2}\right)+\alpha b_{n}\left(\left\|v^{0}\right\|^{2}+\left\|w^{0}\right\|^{2}\right) \\
& =\alpha\left\|v^{0}\right\|^{2}+\alpha\left\|w^{0}\right\|^{2} .
\end{aligned}
$$

This concludes the proof. 


\section{Error estimates for full discretization}

In this section, we discuss fully discrete schemes. Considering different boundary conditions, we choose a Fourier-Galerkin spectral method to discretize semi-discrete scheme (7) and a Legendre-Galerkin method to discretize semi-discrete scheme (8). We present some error estimates for full-discretization schemes in $L^{2}$ norm. First, we define $S_{N}$ to be the Fourier or Legendre polynomial space. Denote $\pi_{N}: L^{2}(\Omega) \rightarrow S_{N}$ to be the $L^{2}$ projection operator which satisfies

$$
\left(\pi_{N} \phi-\phi, \psi\right)=0, \quad \forall \psi \in S_{N}
$$

We also define the $H^{1}$-projection operator $\pi_{N}^{1}: H^{1}(\Omega) \rightarrow S_{N}$ by

$$
\left(\nabla\left(\pi_{N}^{1} \phi-\phi\right), \nabla \psi\right)=0, \quad \forall \psi \in S_{N}
$$

We have the following estimate [37]:

$$
\left\|\phi-\pi_{N} \phi\right\|_{0} \leq C N^{-m}\|\phi\|_{m}, \quad \forall \phi \in H^{m}(\Omega), m \geq 0 .
$$

Consider the full-discretization Fourier-Galerkin/Legendre-Galerkin spectral method to equations (7) and (8) as follows: find $v_{N}^{n+1}, w_{N}^{n+1} \in S_{N}$ such that, for all $\phi_{N}, \psi_{N} \in S_{N}$, they satisfy

$$
\left\{\begin{array}{l}
\left(v_{N}^{n+1}-\sum_{j=0}^{n-1}\left(b_{j}-b_{j+1}\right) v_{N}^{n-j}-b_{n} v_{N}^{0}, \phi_{N}\right)+a_{0}\left(\Delta w^{n+1}, \phi_{N}\right)-a_{0} \beta\left(I_{N} V w^{n+1}, \phi_{N}\right)=0, \\
\left(w_{N}^{n+1}-\sum_{j=0}^{n-1}\left(b_{j}-b_{j+1}\right) w_{N}^{n-j}-b_{n} w_{N}^{0}, \psi_{N}\right)-a_{0}\left(\Delta v_{N}^{n+1}, \psi_{N}\right)+a_{0} \beta\left(I_{N} V v_{N}^{n+1}, \psi_{N}\right)=0,
\end{array}\right.
$$

and

$$
\left\{\begin{array}{l}
\left(v_{N}^{n+1}-\sum_{j=0}^{n-1}\left(b_{j}-b_{j+1}\right) v_{N}^{n-j}-\bar{b}_{n} v_{N}^{0}, \phi_{N}\right)+a_{0}\left(\Delta w^{n+1}, \phi_{N}\right)-a_{0} \beta\left(I_{N} V w^{n+1}, \phi_{N}\right)=0, \\
\left(w_{N}^{n+1}-\sum_{j=0}^{n-1}\left(b_{j}-b_{j+1}\right) w_{N}^{n-j}-\bar{b}_{n} w_{N}^{0}, \psi_{N}\right)-a_{0}\left(\Delta v_{N}^{n+1}, \psi_{N}\right)+a_{0} \beta\left(I_{N} V v_{N}^{n+1}, \psi_{N}\right)=0,
\end{array}\right.
$$

where $I_{N} V$ is the interpolation function of $V$.

We now state the stability results for equations (15) and (16).

Theorem 1 Let $\left(\left\{v_{N}^{n}\right\}_{n=1}^{M-1},\left\{w_{N}^{n}\right\}_{n=1}^{M-1}\right)$ be the numerical solutions of (15), then we derive

$$
\left\|v_{N}^{n+1}\right\|^{2}+\left\|w_{N}^{n+1}\right\|^{2} \leq\left\|v_{N}^{0}\right\|^{2}+\left\|w_{N}^{0}\right\|^{2}
$$

Theorem 2 Let $\left(\left\{v_{N}^{n}\right\}_{n=1}^{M-1},\left\{w_{N}^{n}\right\}_{n=1}^{M-1}\right)$ be the numerical solutions of (16), then we have

$$
\left\|v_{N}^{n+1}\right\|^{2}+\left\|w_{N}^{n+1}\right\|^{2} \leq \alpha\left\|v_{N}^{0}\right\|^{2}+\alpha\left\|w_{N}^{0}\right\|^{2}
$$

Next, we begin to analyze the error estimates of the full-discretization schemes (15) and (16). We denote the truncation error as follows:

$$
r_{1}^{n+1}=\frac{1}{\Gamma(2-\alpha)} \sum_{j=0}^{n} b_{j} \frac{v\left(t_{n+1-j}\right)-v\left(t_{n-j}\right)}{\Delta t^{\alpha}}-\frac{1}{\Gamma(1-\alpha)} \int_{0}^{t_{n+1}} \frac{\partial v(s)}{\partial s} \frac{d s}{\left(t_{n+1}-s\right)^{\alpha}},
$$




$$
r_{2}^{n+1}=\frac{1}{\Gamma(2-\alpha)} \sum_{j=0}^{n} b_{j} \frac{w\left(t_{n+1-j}\right)-w\left(t_{n-j}\right)}{\Delta t^{\alpha}}-\frac{1}{\Gamma(1-\alpha)} \int_{0}^{t_{n+1}} \frac{\partial w(s)}{\partial s} \frac{d s}{\left(t_{n+1}-s\right)^{\alpha}}
$$

From $[29,30]$, we have

$$
\left\|r_{1}^{n+1}\right\| \leq C \Delta t^{2-\alpha}, \quad\left\|r_{2}^{n+1}\right\| \leq C \Delta t^{2-\alpha}
$$

We also define the following error functions:

$$
\begin{array}{ll}
\widetilde{e}_{v}^{n}=\pi_{N} v\left(t_{n}\right)-u_{N}^{n}, \quad \widehat{e}_{v}^{n}=v\left(t_{n}\right)-\pi_{N} v\left(t_{n}\right), \quad e_{v}^{n}=\widehat{e}_{v}^{n}+\widetilde{e}_{v}^{n}=v\left(t_{n}\right)-v_{N}^{n}, \\
\widetilde{e}_{w}^{n}=\pi_{N} w\left(t_{n}\right)-w_{N}^{n}, \quad \widehat{e}_{w}^{n}=w\left(t_{n}\right)-\pi_{N} w\left(t_{n}\right), \quad e_{w}^{n}=\widehat{e}_{w}^{n}+\widetilde{e}_{w}^{n}=w\left(t_{n}\right)-w_{N}^{n} .
\end{array}
$$

The following lemma can help us to analyze the error estimates.

Lemma 4 For $a_{0}, b_{n}, n \geq 0$, we have the following result:

$$
\frac{a_{0}}{b_{n}} \leq 2 \Gamma(1-\alpha) T^{\alpha}
$$

Proof

$$
\begin{aligned}
b_{n} & =\left((n+1)^{1-\alpha}-(n)^{1-\alpha}\right)=n^{1-\alpha}\left(\left(1+\frac{1}{n}\right)^{1-\alpha}-1\right) \\
& =n^{1-\alpha}\left(\frac{(1-a)}{n}+\frac{(1-\alpha)(-\alpha)}{2 !} \frac{1}{n^{2}}+\cdots\right) \\
& \geq n^{1-\alpha}\left(\frac{(1-a)}{n}+\frac{(1-\alpha)(-\alpha)}{2 !} \frac{1}{n^{2}}\right) \\
& =n^{1-\alpha}\left(\frac{(1-a)}{2 n}+\frac{(1-a)}{2 n}+\frac{(1-\alpha)(-\alpha)}{2 !} \frac{1}{n^{2}}\right) .
\end{aligned}
$$

Note that

$$
\frac{(1-a)}{2 n}+\frac{(1-\alpha)(-\alpha)}{2 !} \frac{1}{n^{2}} \geq 0
$$

Therefore, we obtain

$$
\frac{a_{0}}{b_{n}} \leq \frac{2 n^{\alpha} \Delta t^{\alpha} \Gamma(2-\alpha)}{(1-\alpha)} \leq 2 \Gamma(1-\alpha) T^{\alpha}
$$

We show the error estimate of full-discretization problem (15) in the following theorem.

Theorem 3 Suppose that $u=v+i w$ is the exact solution of $(1)-(3),(v, w)$ and $\left(\left\{v_{N}^{n}\right\}_{n=1}^{M-1}\right.$, $\left.\left\{v_{N}^{n}\right\}_{n=1}^{M-1}\right)$ are the solutions of (6) and (15), respectively, then we have

$$
\left\|v\left(\cdot, t_{n}\right)-v_{N}^{n}\right\|+\left\|w\left(\cdot, t_{n}\right)-w_{N}^{n}\right\| \leq C\left(\Delta t^{2-\alpha}+N^{-s}+N^{-m}\right),
$$

where $C$ depends only on $u, V, T, \alpha, \beta$. 
Proof We will utilize mathematical induction to prove the above conclusion. For $n=0$, equation (15) can be written as

$$
\left\{\begin{array}{l}
\frac{1}{a_{0}}\left(v_{N}^{1}-v_{N}^{0}, \phi_{N}\right)+\left(\Delta w_{N}^{1}, \phi_{N}\right)-\beta\left(I_{N} V w_{N}^{1}, \phi_{N}\right)=0, \\
\frac{1}{a_{0}}\left(w_{N}^{1}-w_{N}^{0}, \psi_{N}\right)-\left(\Delta v_{N}^{1}, \psi_{N}\right)+\beta\left(I_{N} V v_{N}^{1}, \psi_{N}\right)=0 .
\end{array}\right.
$$

Subtracting (24) from a reformulation of (6) at $t_{1}$, we obtain

$$
\begin{aligned}
\left(\widetilde{e}_{v}^{1}\right. & \left.-\widetilde{e}_{v}^{0}, \phi_{N}\right)+a_{0}\left(\Delta \widetilde{e}_{w}^{1}, \phi_{N}\right)-a_{0} \beta\left(V w\left(t_{1}\right)-I_{N} V w_{N}^{1}, \phi_{N}\right) \\
& =a_{0}\left(r_{1}^{1}, \phi_{N}\right)+\left(\left(\pi_{N}-I\right)\left(v\left(t_{1}\right)-v\left(t_{0}\right)\right), \phi_{N}\right)-a_{0}\left(\nabla\left(\pi_{N}^{1}-I\right) w\left(t_{1}\right), \nabla \phi_{N}\right), \\
\left(\widetilde{e}_{w}^{1}-\widetilde{e}_{w}^{0}, \psi_{N}\right)-a_{0}\left(\Delta \widetilde{e}_{v}^{1}, \psi_{N}\right)+a_{0} \beta\left(V v\left(t_{1}\right)-I_{N} V v_{N}^{1}, \psi_{N}\right) & \\
= & a_{0}\left(r_{2}^{1}, \psi_{N}\right)+\left(\left(\pi_{N}-I\right)\left(w\left(t_{1}\right)-w\left(t_{0}\right)\right), \psi_{N}\right)+a_{0}\left(\nabla\left(\pi_{N}^{1}-I\right) v\left(t_{1}\right), \nabla \psi_{N}\right) .
\end{aligned}
$$

Let $\phi_{N}=2 \widetilde{e}_{v}^{1}, \psi_{N}=2 \widetilde{e}_{w}^{1}$, we have

$$
\begin{aligned}
2\left\|\widetilde{e}_{v}^{1}\right\|^{2}+2\left\|\widetilde{e}_{w}^{1}\right\|^{2} \leq & 2 a_{0}\left(r_{1}^{1}, \widetilde{e}_{v}^{1}\right)+2 a_{0}\left(r_{2}^{1}, \widetilde{e}_{w}^{1}\right) \\
& +2\left(\left(\pi_{N}-I\right)\left(v\left(t_{1}\right)-v\left(t_{0}\right)\right), \widetilde{e}_{v}^{1}\right)+2\left(\left(\pi_{N}-I\right)\left(w\left(t_{1}\right)-w\left(t_{0}\right)\right), \widetilde{e}_{w}^{1}\right) \\
& +2 a_{0} \beta\left(w\left(t_{1}\right)\left(V-I_{N} V\right)+I_{N} V\left(w\left(t_{1}\right)-w_{N}^{1}\right), \widetilde{e}_{v}^{1}\right) \\
& -2 a_{0} \beta\left(v\left(t_{1}\right)\left(V-I_{N} V\right)+I_{N} V\left(v\left(t_{1}\right)-v_{N}^{1}\right), \widetilde{e}_{w}^{1}\right) .
\end{aligned}
$$

Note that

$$
\begin{aligned}
2 a_{0}\left(w\left(t_{1}\right)\left(V-I_{N} V\right)+I_{N} V\left(w\left(t_{1}\right)-w_{N}^{1}\right), \widetilde{e}_{v}^{1}\right) & \\
& -2 a_{0}\left(v\left(t_{1}\right)\left(V-I_{N} V\right)+I_{N} V\left(v\left(t_{1}\right)-v_{N}^{1}\right), \widetilde{e}_{w}^{1}\right) \\
= & 2 a_{0}\left(w\left(t_{1}\right)\left(V-I_{N} V\right)+I_{N} V\left(\widetilde{e}_{w}^{1}+\widehat{e}_{w}^{1}\right), \widetilde{e}_{v}^{1}\right)-2 a_{0}\left(v\left(t_{1}\right)\left(V-I_{N} V\right)+I_{N} V\left(\widetilde{e}_{v}^{1}+\widehat{e}_{v}^{1}\right), \widetilde{e}_{w}^{1}\right) \\
= & 2 a_{0}\left(w\left(t_{1}\right)\left(V-I_{N} V\right)+I_{N} V \widehat{e}_{w}^{1}, \widetilde{e}_{v}^{1}\right)-2 a_{0}\left(v\left(t_{1}\right)\left(V-I_{N} V\right)+I_{N} V \widehat{e}_{v}^{1}, \widetilde{e}_{w}^{1}\right) .
\end{aligned}
$$

Using Young's inequality, we get

$$
\begin{aligned}
2\left\|\widetilde{e}_{v}^{1}\right\|^{2}+2\left\|\widetilde{e}_{w}^{1}\right\|^{2} & \leq a_{0}\left(4 a_{0}\left\|r_{1}^{1}\right\|^{2}+\frac{1}{4 a_{0}}\left\|\widetilde{e}_{v}^{1}\right\|^{2}\right)+a_{0}\left(4 a_{0}\left\|r_{2}^{1}\right\|^{2}+\frac{1}{4 a_{0}}\left\|\widetilde{e}_{w}^{1}\right\|^{2}\right) \\
& +4\left\|\left(\pi_{N}-I\right)\left(v\left(t_{1}\right)-v\left(t_{0}\right)\right)\right\|^{2}+\frac{1}{4}\left\|\widetilde{e}_{v}^{1}\right\|^{2}+4\left\|\left(\pi_{N}-I\right)\left(w\left(t_{1}\right)-w\left(t_{0}\right)\right)\right\|^{2}+\frac{1}{4}\left\|\widetilde{e}_{w}^{1}\right\|^{2} \\
& +a_{0} \beta\left(4 a_{0} \beta\left\|I_{N} V \widehat{e}_{w}^{1}\right\|^{2}+\frac{1}{4 a_{0} \beta}\left\|\widetilde{e}_{v}^{1}\right\|^{2}\right) \\
& +a_{0} \beta\left(4 a_{0} \beta\left\|w\left(t_{1}\right)\left(V-I_{N} V\right)\right\|^{2}+\frac{1}{4 a_{0} \beta}\left\|\widetilde{e}_{v}^{1}\right\|^{2}\right) \\
& +a_{0} \beta\left(4 a_{0} \beta\left\|I_{N} V \widehat{e}_{v}^{1}\right\|^{2}+\frac{1}{4 a_{0} \beta}\left\|\widetilde{e}_{w}^{1}\right\|^{2}\right) \\
& +a_{0} \beta\left(4 a_{0} \beta\left\|v\left(t_{1}\right)\left(V-I_{N} V\right)\right\|^{2}+\frac{1}{4 a_{0} \beta}\left\|\widetilde{e}_{w}^{1}\right\|^{2}\right)
\end{aligned}
$$


Chang et al. Advances in Difference Equations

(2020) 2020:53

Page 10 of 16

$$
\begin{aligned}
\leq & 4 a_{0}^{2}\left(\left\|r_{1}\right\|^{2}+\left\|r_{2}\right\|^{2}\right)+4\left\|\left(\pi_{N}-I\right)\left(v\left(t_{1}\right)-v\left(t_{0}\right)\right)\right\|^{2}+4\left\|\left(\pi_{N}-I\right)\left(w\left(t_{1}\right)-w\left(t_{0}\right)\right)\right\|^{2} \\
& +4 a_{0}^{2} \beta^{2}\left\|I_{N} V\right\|_{\infty}\left\|\widehat{e}_{w}^{1}\right\|^{2}+4 a_{0}^{2} \beta^{2}\left\|w\left(t_{1}\right)\right\|_{\infty}\left\|V-I_{N} V\right\|^{2}+\left\|\widetilde{e}_{w}^{1}\right\|^{2}+\left\|\widetilde{e}_{v}^{1}\right\|^{2} \\
& +4 a_{0}^{2} \beta^{2}\left\|I_{N} V\right\|_{\infty}\left\|\widehat{e}_{v}^{1}\right\|^{2}+4 a_{0}^{2} \beta^{2}\left\|v\left(t_{1}\right)\right\|_{\infty}\left\|V-I_{N} V\right\|^{2} .
\end{aligned}
$$

That is

$$
\left\|\widetilde{e}_{v}^{1}\right\|^{2}+\left\|\widetilde{e}_{w}^{1}\right\|^{2} \leq C_{1} a_{0}^{2} \Delta t^{4-2 \alpha}+C_{2} a_{0}^{2} N^{-s}+C_{3} a_{0}^{2} N^{-m}
$$

Assume

$$
\left\|\widetilde{e}_{v}^{j}\right\|^{2}+\left\|\widetilde{e}_{w}^{j}\right\|^{2} \leq \frac{C_{1} a_{0}^{2} \Delta t^{4-2 \alpha}}{b_{j-1}^{2}}+\frac{C_{2} a_{0}^{2} N^{-s}}{b_{j-1}^{2}}+\frac{C_{3} a_{0}^{2} N^{-m}}{b_{j-1}^{2}}, \quad j=2,3, \ldots, n .
$$

Next, we need to prove that it also holds for $j=n+1$. Setting $\phi_{N}=2 \widetilde{e}_{v}^{n+1}$ and $\psi_{N}=2 \widetilde{e}_{w}^{n+1}$ in (15), we have

$$
\begin{aligned}
& 2\left\|\widetilde{e}_{v}^{n+1}\right\|^{2}+2\left\|\widetilde{e}_{w}^{n+1}\right\|^{2} \\
& \leq 2 a_{0}\left(r_{1}^{n+1}, \widetilde{e}_{v}^{n+1}\right)+2 a_{0}\left(r_{2}^{n+1}, \widetilde{e}_{w}^{n+1}\right) \\
& +2\left(\sum_{j=0}^{n-1}\left(b_{j}-b_{j+1}\right) \widetilde{e}_{v}^{n-j}+b_{n} \widetilde{e}_{v}^{0}, \widetilde{e}_{v}^{n+1}\right)+2\left(\sum_{j=0}^{n-1}\left(b_{j}-b_{j+1}\right) \widetilde{e}_{w}^{n-j}+b_{n} \widetilde{e}_{w}^{0}, \widetilde{e}_{w}^{n+1}\right) \\
& +2\left(\left(\pi_{N}-I\right)\left(v\left(t_{n+1}\right)-\sum_{j=0}^{n}\left(b_{j}-b_{j+1}\right) v\left(t_{n-j}\right)-b_{n} v\left(t_{0}\right)\right), \widetilde{e}_{v}^{n+1}\right) \\
& +2\left(\left(\pi_{N}-I\right)\left(w\left(t_{n+1}\right)-\sum_{j=0}^{n}\left(b_{j}-b_{j+1}\right) w\left(t_{n-j}\right)-b_{n} w\left(t_{0}\right)\right), \widetilde{e}_{w}^{n+1}\right) \\
& +2 a_{0} \beta\left(w\left(t_{n+1}\right)\left(V-I_{N} V\right)+I_{N} V\left(w\left(t_{n+1}\right)-w_{N}^{n+1}\right), \widetilde{e}_{v}^{n+1}\right) \\
& -2 a_{0} \beta\left(v\left(t_{n+1}\right)\left(V-I_{N} V\right)+I_{N} V\left(v\left(t_{n+1}\right)-v_{N}^{n+1}\right), \widetilde{e}_{w}^{n+1}\right) \\
& \leq a_{0}\left(\frac{4 a_{0}}{b_{n}}\left\|r_{1}^{n+1}\right\|^{2}+\frac{b_{n}}{4 a_{0}}\left\|\widetilde{e}_{v}^{n+1}\right\|^{2}\right)+a_{0}\left(\frac{4 a_{0}}{b_{n}}\left\|r_{2}^{n+1}\right\|^{2}+\frac{b_{n}}{4 a_{0}}\left\|\widetilde{e}_{w}^{n+1}\right\|^{2}\right) \\
& +\sum_{j=0}^{n-1}\left(b_{j}-b_{j+1}\right)\left(\left\|\widetilde{e}_{v}^{n-j}\right\|^{2}+\left\|\widetilde{e}_{v}^{n+1}\right\|^{2}\right)+\sum_{j=0}^{n-1}\left(b_{j}-b_{j+1}\right)\left(\left\|\widetilde{e}_{w}^{n-j}\right\|^{2}+\left\|\widetilde{e}_{w}^{n+1}\right\|^{2}\right) \\
& +\frac{4}{b_{n}}\left\|\left(\pi_{N}-I\right)\left(v\left(t_{n+1}\right)-\sum_{j=0}^{n}\left(b_{j}-b_{j+1}\right) v\left(t_{n-j}\right)-b_{n} v\left(t_{0}\right)\right)\right\|^{2}+\frac{b_{n}}{4}\left\|\widetilde{e}_{v}^{n+1}\right\|^{2} \\
& +\frac{4}{b_{n}}\left\|\left(\pi_{N}-I\right)\left(w\left(t_{n+1}\right)-\sum_{j=0}^{n}\left(b_{j}-b_{j+1}\right) w\left(t_{n-j}\right)-b_{n} v\left(t_{0}\right)\right)\right\|^{2}+\frac{b_{n}}{4}\left\|\widetilde{e}_{w}^{n+1}\right\|^{2} \\
& +a_{0} \beta\left(\frac{4 a_{0} \beta}{b_{n}}\left\|I_{N} V \widehat{e}_{w}^{n+1}\right\|^{2}+\frac{b_{n}}{4 a_{0} \beta}\left\|\widetilde{e}_{v}^{n+1}\right\|^{2}\right) \\
& +a_{0} \beta\left(\frac{4 a_{0} \beta}{b_{n}}\left\|w\left(t_{n+1}\right)\left(V-I_{N} V\right)\right\|^{2}+\frac{b_{n}}{4 a_{0} \beta}\left\|\widetilde{e}_{v}^{n+1}\right\|^{2}\right) \\
& +a_{0} \beta\left(\frac{4 a_{0} \beta}{b_{n}}\left\|I_{N} V \widehat{e}_{v}^{n+1}\right\|^{2}+\frac{b_{n}}{4 a_{0} \beta}\left\|\widetilde{e}_{w}^{n+1}\right\|^{2}\right)
\end{aligned}
$$




$$
+a_{0} \beta\left(\frac{4 a_{0} \beta}{b_{n}}\left\|v\left(t_{n+1}\right)\left(V-I_{N} V\right)\right\|^{2}+\frac{b_{n}}{4 a_{0} \beta}\left\|\widetilde{e}_{w}^{n+1}\right\|^{2}\right)
$$

That is

$$
\begin{aligned}
\left\|\widetilde{e}_{v}^{n+1}\right\|^{2}+\left\|\widetilde{e}_{w}^{n+1}\right\|^{2} \leq & \frac{4 a_{0}^{2}}{b_{n}}\left(\left\|r_{1}^{n+1}\right\|^{2}+\left\|r_{2}^{n+1}\right\|^{2}\right)+\sum_{j=0}^{n-1}\left(b_{j}-b_{j+1}\right)\left(\left\|\widetilde{e}_{v}^{n-j}\right\|^{2}+\left\|\widetilde{e}_{w}^{n-j}\right\|^{2}\right) \\
& +\frac{4}{b_{n}}\left\|\left(\pi_{N}-I\right)\left(v\left(t_{n+1}\right)-\sum_{j=0}^{n}\left(b_{j}-b_{j+1}\right) v\left(t_{n-j}\right)-b_{n} v\left(t_{0}\right)\right)\right\|^{2} \\
& +\frac{4}{b_{n}}\left\|\left(\pi_{N}-I\right)\left(w\left(t_{n+1}\right)-\sum_{j=0}^{n}\left(b_{j}-b_{j+1}\right) w\left(t_{n-j}\right)-b_{n} w\left(t_{0}\right)\right)\right\|^{2} \|^{2} \\
& +\frac{4 a_{0}^{2} \beta^{2}}{b_{n}}\left\|I_{N} V\right\|_{\infty}\left\|\widehat{e}_{w}^{n+1}\right\|^{2}+\frac{4 a_{0}^{2} \beta^{2}}{b_{n}}\left\|w\left(t_{n+1}\right)\right\|_{\infty}\left\|V-I_{N} V\right\|^{2} \\
& +\frac{4 a_{0}^{2} \beta^{2}}{b_{n}}\left\|I_{N} V\right\|_{\infty}\left\|\widehat{e}_{v}^{n+1}\right\|^{2}+\frac{4 a_{0}^{2} \beta^{2}}{b_{n}}\left\|v\left(t_{n+1}\right)\right\|_{\infty}\left\|V-I_{N} V\right\|^{2} .
\end{aligned}
$$

Using assumption (25) and the fact that $b_{n-1-j}>b_{n}$, we obtain

$$
\begin{aligned}
\left\|\widetilde{e}_{v}^{n+1}\right\|^{2}+\left\|\widetilde{e}_{w}^{n+1}\right\|^{2} \leq & \left(\frac{C_{1} a_{0}^{2} \Delta t^{4-2 \alpha}}{b_{n}}+\frac{C_{2} a_{0}^{2} N^{-s}}{b_{n}}+\frac{C_{3} a_{0}^{2} N^{-m}}{b_{n}}\right) \\
& +\sum_{j=0}^{n-1}\left(b_{j}-b_{j+1}\right)\left(\frac{C_{1} a_{0}^{2} \Delta t^{4-2 \alpha}}{b_{n-j-1}^{2}}+\frac{C_{2} a_{0}^{2} N^{-s}}{b_{n-j-1}^{2}}+\frac{C_{3} a_{0}^{2} N^{-m}}{b_{n-j-1}^{2}}\right) \\
\leq & \left(\frac{C_{1} a_{0}^{2} \Delta t^{4-2 \alpha}}{b_{n}}+\frac{C_{2} a_{0}^{2} N^{-s}}{b_{n}}+\frac{C_{3} a_{0}^{2} N^{-m}}{b_{n}}\right) \\
& +\sum_{j=0}^{n-1}\left(b_{j}-b_{j+1}\right)\left(\frac{C_{1} a_{0}^{2} \Delta t^{4-2 \alpha}}{b_{n}^{2}}+\frac{C_{2} a_{0}^{2} N^{-s}}{b_{n}^{2}}+\frac{C_{3} a_{0}^{2} N^{-m}}{b_{n}^{2}}\right) \\
= & \left(\frac{C_{1} a_{0}^{2} \Delta t^{4-2 \alpha}}{b_{n}^{2}}+\frac{C_{2} a_{0}^{2} N^{-s}}{b_{n}^{2}}+\frac{C_{3} a_{0}^{2} N^{-m}}{b_{n}^{2}}\right)\left(b_{n}+\sum_{j=0}^{n-1}\left(b_{j}-b_{j+1}\right)\right) \\
= & \left(\frac{C_{1} a_{0}^{2} \Delta t^{4-2 \alpha}}{b_{n}^{2}}+\frac{C_{2} a_{0}^{2} N^{-s}}{b_{n}^{2}}+\frac{C_{3} a_{0}^{2} N^{-m}}{b_{n}^{2}}\right) .
\end{aligned}
$$

Combining with (14) and (22), estimate (23) is proved.

Theorem 4 Suppose that $u=v+i w$ is the exact solution of $(1)-(3),(v, w)$ and $\left(\left\{v_{N}^{n}\right\}_{n=1}^{M-1}\right.$, $\left.\left\{v_{N}^{n}\right\}_{n=1}^{M-1}\right)$ are the solutions to (6) and (16), respectively, then we obtain

$$
\left\|v\left(\cdot, t_{n}\right)-v_{N}^{n}\right\|+\left\|w\left(\cdot, t_{n}\right)-w_{N}^{n}\right\| \leq C\left(\Delta t^{2-\alpha}+N^{-s}+N^{-m}\right) .
$$

Proof The proof process is similar to the above theorem, and we omit it here.

\section{Numerical results}

This section presents several numerical examples to confirm the accuracy and applicability of schemes (15)-(16) for solving Caputo/Riemann-Liouville Schrödinger equations. First, we need an exact solution to evaluate the accuracy of the numerical solution. 
Example 4.1 Let $\beta=1$ and $V=1$, we consider numerical results for the following Caputo time-fractional Schrödinger equation:

$$
\frac{1}{\Gamma(1-\alpha)} \int_{0}^{t} \frac{\partial u(x, y, \mu)}{\partial \mu} \frac{d \mu}{(t-\mu)^{\alpha}}-i \Delta u+i u=f(x, y, t)
$$

where

$$
f(x, y, t)=\frac{2 t^{2-\alpha}}{\Gamma(3-\alpha)}(\cos 8 x+i \sin 8 y)+t^{2}(-65 \sin 8 y+i 65 \cos 8 x) .
$$

Then the exact solution is $u=t^{2}(\cos 8 x+i \sin 8 y)$.

Full-discrete scheme (15) is solved in $\Omega=(0,2 \pi)^{2}$ with $T=1$. Tables $1-2$ display the temporal convergence orders at $N=20$ for $v, w$, respectively. It shows that for $\alpha=$ $0.1,0.3,0.5,0.6,0.7,0.9$, the convergence orders of $v$ and $w$ are approximately $1.9,1.7,1.5$, $1.4,1.3,1.1$, respectively, which indicates that numerical scheme (15) can achieve $2-\alpha$ order accuracy in time, which confirms the result in Theorem 3. Table 3 shows the $L^{2}$ error and the $L^{\infty}$ error in space with $\alpha=0.1,0.3,0.5,0.7$. It confirms that the numerical solutions are in good agreement with the exact solutions and the error is influenced by the time direction error. It ascertains that, if the error in the time direction is negligible, our numerical method can theoretically achieve exponential order accuracy in space.

Table 1 Temporal convergence orders of $v$ for Example 4.1

\begin{tabular}{lllll}
\hline$\alpha \backslash \Delta t$ & $\Delta t=1.00 \mathrm{E}-2$ & $\Delta t=5.00 \mathrm{E}-3$ & $\Delta t=1.00 \mathrm{E}-3$ & $\Delta t=5.00 \mathrm{E}-4$ \\
\hline$\alpha=0.1$ & 1.8015 & 1.8138 & 1.8349 & 1.8418 \\
$\alpha=0.3$ & 1.6588 & 1.6674 & 1.6807 & 1.6845 \\
$\alpha=0.5$ & 1.4857 & 1.4900 & 1.4956 & 1.4969 \\
$\alpha=0.6$ & 1.3923 & 1.3949 & 1.3981 & 1.3987 \\
$\alpha=0.7$ & 1.2962 & 1.2976 & 1.2992 & 1.2995 \\
$\alpha=0.9$ & 1.0995 & 1.0997 & 1.0999 & 1.1000
\end{tabular}

Table 2 Temporal convergence orders of $w$ for Example 4.1

\begin{tabular}{lllll}
\hline$\alpha \backslash \Delta t$ & $\Delta t=1.00 \mathrm{E}-2$ & $\Delta t=5.00 \mathrm{E}-3$ & $\Delta t=1.00 \mathrm{E}-3$ & $\Delta t=5.00 \mathrm{E}-4$ \\
\hline$\alpha=0.1$ & 1.8015 & 1.8138 & 1.8349 & 1.8418 \\
$\alpha=0.3$ & 1.6588 & 1.6674 & 1.6807 & 1.6845 \\
$\alpha=0.5$ & 1.4857 & 1.4900 & 1.4956 & 1.4969 \\
$\alpha=0.6$ & 1.3923 & 1.3949 & 1.3981 & 1.3987 \\
$\alpha=0.7$ & 1.2961 & 1.2976 & 1.2992 & 1.2995 \\
$\alpha=0.9$ & 1.0994 & 1.0997 & 1.0999 & 1.1000 \\
\hline
\end{tabular}

Table $3 L^{2}, L^{\infty}$ errors of $v$ and $w$ at $\Delta t=10^{-4}$ for Example 4.1

\begin{tabular}{llllll}
\hline$\alpha \backslash$ error & $N$ & $\left\|v(T)-v_{N}^{M}\right\|$ & $\left\|v(T)-v_{N}^{M}\right\|_{\infty}$ & $\left\|w(T)-w_{N}^{M}\right\|$ & $\left\|w(T)-w_{N}^{M}\right\|_{\infty}$ \\
\hline$\alpha=0.1$ & 18 & $1.7195 \mathrm{E}-10$ & $5.6609 \mathrm{E}-10$ & $1.7196 \mathrm{E}-10$ & $5.7301 \mathrm{E}-10$ \\
& 20 & $1.8126 \mathrm{E}-10$ & $5.4817 \mathrm{E}-11$ & $1.8125 \mathrm{E}-10$ & $5.7301 \mathrm{E}-11$ \\
$\alpha=0.3$ & 18 & $2.2952 \mathrm{E}-09$ & $7.5500 \mathrm{E}-10$ & $2.2952 \mathrm{E}-09$ & $7.6500 \mathrm{E}-10$ \\
& 20 & $2.4193 \mathrm{E}-09$ & $7.3044 \mathrm{E}-10$ & $1.8125 \mathrm{E}-10$ & $5.7301 \mathrm{E}-11$ \\
$\alpha=0.5$ & 18 & $2.1610 \mathrm{E}-08$ & $7.1047 \mathrm{E}-10$ & $2.1610 \mathrm{E}-08$ & $7.2032 \mathrm{E}-09$ \\
& 20 & $2.2779 \mathrm{E}-08$ & $6.8701 \mathrm{E}-09$ & $2.2779 \mathrm{E}-08$ & $7.2032 \mathrm{E}-09$ \\
$\alpha=0.7$ & 18 & $1.9066 \mathrm{E}-07$ & $6.2644 \mathrm{E}-08$ & $1.9066 \mathrm{E}-07$ & $6.3553 \mathrm{E}-08$ \\
& 20 & $2.0098 \mathrm{E}-07$ & $6.0544 \mathrm{E}-08$ & $2.0098 \mathrm{E}-07$ & $6.3553 \mathrm{E}-08$ \\
\hline
\end{tabular}


Example 4.2 Let $\beta=1$ and $V=1$, we consider the following Riemann-Liouville timefractional Schrödinger equation:

$$
\frac{1}{\Gamma(1-\alpha)} \frac{d}{d t} \int_{0}^{t}(t-\mu)^{-\alpha} u(x, y, \mu) d \mu-i \Delta u+i u=f(x, y, t)
$$

where

$$
f(x, y, t)=\left(\frac{t^{-\alpha}}{\Gamma(1-\alpha)}+\frac{2 t^{2-\alpha}}{\Gamma(3-\alpha)}\right)(\sin 8 x+i \cos 8 y)+\left(t^{2}+1\right)(-65 \cos 8 y+i 65 \sin 8 x) .
$$

Then the exact solution is $u=\left(t^{2}+1\right)(\sin 8 x+i \cos 8 y)$.

Full-discrete scheme (16) is solved at $\Omega=(0,2 \pi)^{2}$ with $T=1$. The convergence orders in time direction are listed in Tables $4-5$, and the $L^{2}$ and $L^{\infty}$ errors in spatial direction are also listed in Table 6 . It is obvious that numerical scheme (16) achieves $2-\alpha$ order accuracy in time direction. Table 6 shows that numerical schemes (15)-(16) have good convergence behavior.

We fix $u(x, y, 0)=\cos 6 x+i \sin y, \Delta t=0.001, N=20$, we also use numerical method (15) to simulate the dynamic behavior of the solution for different $\alpha$. The results are summarized in Figs. 1-3. The results show that the wavelength becomes larger when the fractional diffusion coefficient $\alpha$ increases due to the long tail mechanism of the fractional operator.

Table 4 Temporal convergence orders of $v$ for Example 4.2

\begin{tabular}{lllll}
\hline$\alpha \backslash \Delta t$ & $\Delta t=1.00 \mathrm{E}-2$ & $\Delta t=5.00 \mathrm{E}-3$ & $\Delta t=1.00 \mathrm{E}-3$ & $\Delta t=5.00 \mathrm{E}-4$ \\
\hline$\alpha=0.1$ & 1.8015 & 1.8138 & 1.8349 & 1.8418 \\
$\alpha=0.3$ & 1.6588 & 1.6674 & 1.6807 & 1.6845 \\
$\alpha=0.5$ & 1.4857 & 1.4900 & 1.4956 & 1.4969 \\
$\alpha=0.6$ & 1.3923 & 1.3949 & 1.3981 & 1.3987 \\
$\alpha=0.7$ & 1.2961 & 1.2976 & 1.2992 & 1.2995 \\
$\alpha=0.9$ & 1.0994 & 1.0997 & 1.0999 & 1.1000 \\
\hline
\end{tabular}

Table 5 Temporal convergence orders of $w$ for Example 4.2

\begin{tabular}{lllll}
\hline$\alpha \backslash \Delta t$ & $\Delta t=1.00 \mathrm{E}-2$ & $\Delta t=5.00 \mathrm{E}-3$ & $\Delta t=1.00 \mathrm{E}-3$ & $\Delta t=5.00 \mathrm{E}-4$ \\
\hline$\alpha=0.1$ & 1.8015 & 1.8138 & 1.8349 & 1.8418 \\
$\alpha=0.3$ & 1.6588 & 1.6674 & 1.6807 & 1.6845 \\
$\alpha=0.5$ & 1.4857 & 1.4900 & 1.4956 & 1.4969 \\
$\alpha=0.6$ & 1.3923 & 1.3949 & 1.3981 & 1.3987 \\
$\alpha=0.7$ & 1.2961 & 1.2976 & 1.2992 & 1.2995 \\
$\alpha=0.9$ & 1.0994 & 1.0997 & 1.0999 & 1.1000 \\
\hline
\end{tabular}

Table $6 L^{2}, L^{\infty}$ errors of $v$ and $w$ at $\Delta t=10^{-4}$ for Example 4.2

\begin{tabular}{llllll}
\hline$\alpha \backslash \Delta t$ & $N$ & $\left\|v(T)-v_{N}^{M}\right\|$ & $\left\|v(T)-v_{N}^{M}\right\|_{\infty}$ & $\left\|w(T)-w_{N}^{M}\right\|$ & $\left\|w(T)-w_{N}^{M}\right\|_{\infty}$ \\
\hline$\alpha=0.1$ & 18 & $1.7193 \mathrm{E}-10$ & $5.7337 \mathrm{E}-11$ & $1.7195 \mathrm{E}-10$ & $5.6706 \mathrm{E}-11$ \\
& 20 & $1.8124 \mathrm{E}-10$ & $5.7430 \mathrm{E}-11$ & $1.8124 \mathrm{E}-10$ & $5.4762 \mathrm{E}-11$ \\
$\alpha=0.3$ & 18 & $2.2951 \mathrm{E}-09$ & $7.6502 \mathrm{E}-10$ & $2.2952 \mathrm{E}-09$ & $7.5509 \mathrm{E}-10$ \\
& 20 & $2.4193 \mathrm{E}-09$ & $7.6511 \mathrm{E}-10$ & $2.4193 \mathrm{E}-09$ & $7.3037 \mathrm{E}-10$ \\
$\alpha=0.5$ & 18 & $2.1610 \mathrm{E}-08$ & $7.2032 \mathrm{E}-09$ & $2.1610 \mathrm{E}-08$ & $7.1048 \mathrm{E}-09$ \\
& 20 & $2.2779 \mathrm{E}-08$ & $7.2033 \mathrm{E}-09$ & $2.2779 \mathrm{E}-08$ & $6.8700 \mathrm{E}-09$ \\
$\alpha=0.7$ & 18 & $1.9066 \mathrm{E}-07$ & $6.3553 \mathrm{E}-08$ & $1.9066 \mathrm{E}-07$ & $6.2645 \mathrm{E}-08$ \\
& 20 & $2.0098 \mathrm{E}-07$ & $6.3553 \mathrm{E}-08$ & $2.0098 \mathrm{E}-07$ & $6.0544 \mathrm{E}-08$ \\
\hline
\end{tabular}




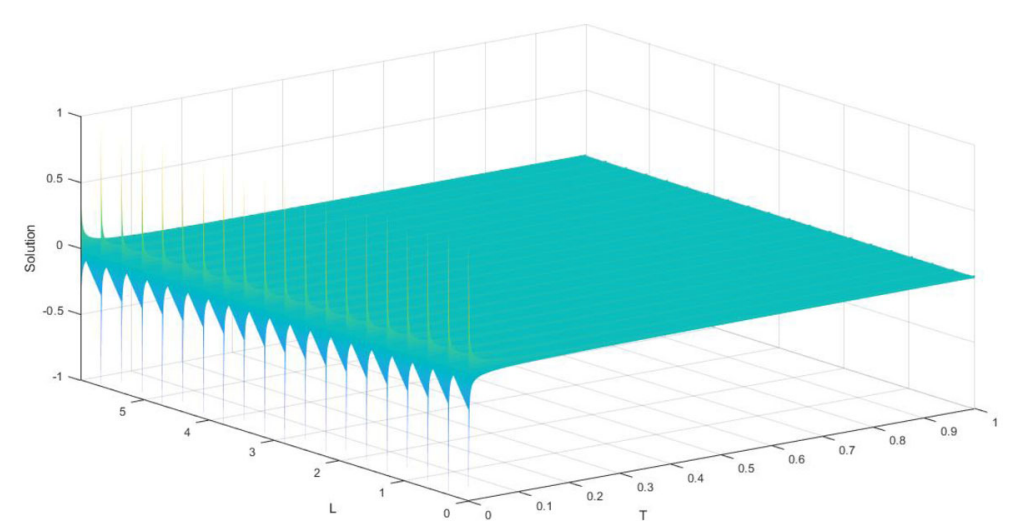

Figure 1 The dynamic behavior of solution for $\alpha=0.5$

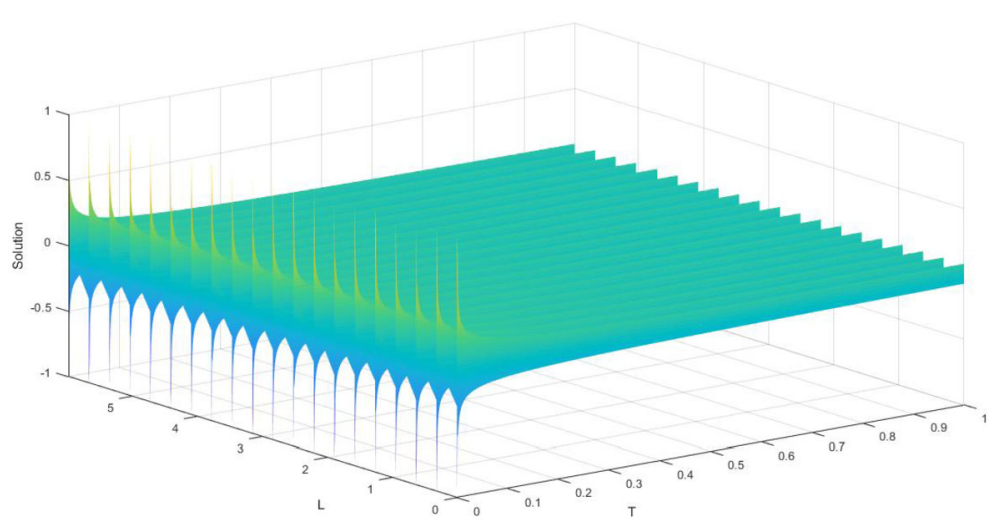

Figure 2 The dynamic behavior of solution for $\alpha=0.7$

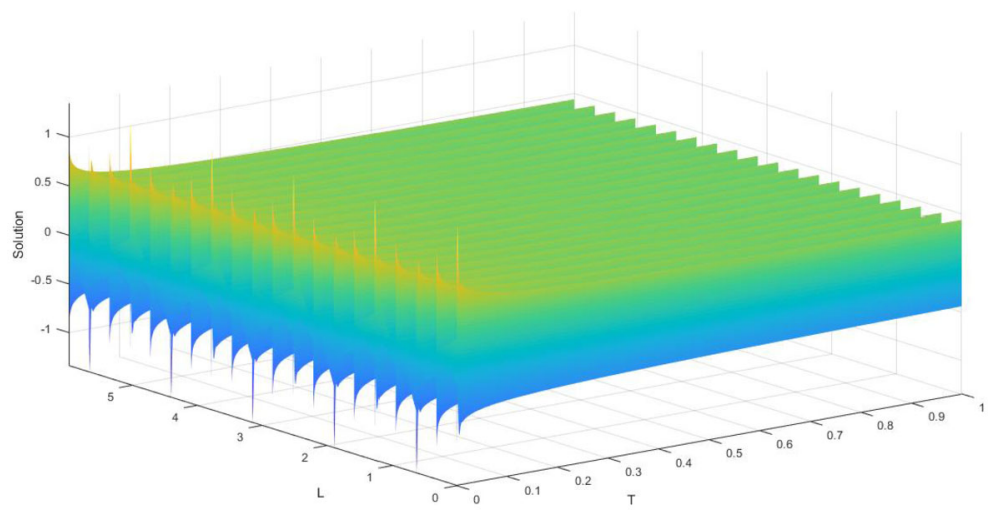

Figure 3 The dynamic behavior of solution for $\alpha=0.9$

\section{Conclusion}

We have constructed two efficient numerical schemes to solve time-fractional Schrödinger equation with Caputo/Riemann-Liouville derivative. These two numerical methods have been proved to be unconditionally stable. In addition, we have also discussed the conver- 
gence of the numerical methods, and numerical convergence results show that the two schemes converge with the order $\mathcal{O}\left(\Delta t^{2-\alpha}+N^{-s}+N^{-m}\right)$. Numerical examples are consistent with the theoretical prediction.

\section{Acknowledgements}

The authors would like to thank editors and reviewers for their help.

\section{Funding}

The work of Jun Zhang is supported by the National Natural Science Foundation of China, Grant/Award Number: 11901132,61472093; the China Scholarship Council, Grant/Award Number: 201908525061; the Chinese Postdoc Foundation, Grant Grant/Award Number: 2019M653490, and Guizhou Province University science and technology top talents project, Grant/Award Number: KY[2018]047. The work of JinRong Wang is supported by the National Natural Science Foundation of China, Grant/Award Number: 11661016; Training Object of High Level and Innovative Talents of Guizhou Province, Grant/Award Number: (2016)4006; Major Research Project of Innovative Group in Guizhou Education Department, Grant/Award Number: [2018]012. The work of Yong Zhou is supported by the National Natural Science Foundation of China, Grant/Award Number: 11671339

\section{Availability of data and materials}

Data sharing not applicable to this article as no datasets were generated or analyzed during the current study.

\section{Ethics approval and consent to participate}

Not applicable.

\section{Competing interests}

The authors declare that they have no competing interests.

Consent for publication

We agree.

Authors' contributions

JZ carried out an efficient numerical approach to solve time-fractional Schrödinger. JRW helped to draft the manuscript. $Y Z$ helped to correct some typos and grammar errors. All authors read and approved the final manuscript.

\section{Authors' information}

Jun Zhang, Computational Mathematics Research Center, Guizhou University of Finance and Economics, Guiyang 550025, Department of Mathematics, Guizhou University, Guiyang, Guizhou 550025, China. E-mail addresses: jzhang@mail.gufe.edu.cn. JingRong Wang, Corresponding author, Department of Mathematics, Guizhou University, Guiyang, 550025, China and School of Mathematical Sciences, Qufu Normal University, Qufu 273165, Shandong, China. E-mail addresses: jrwang@gzu.edu.cn. Yong Zhou, Department of Mathematics, Xiangtan University, Xiangtan, Hunan 411105, China and Faculty of Information Technology, Macau University of Science and Technology, Taipa, China. E-mail addresses: yzhou@xtu.edu.cn.

\section{Author details}

${ }^{1}$ Department of Mathematics, Guizhou University, Guiyang, China. ${ }^{2}$ Computational Mathematics Research Center, Guizhou University of Finance and Economics, Guiyang, China. ${ }^{3}$ School of Mathematical Sciences, Qufu Normal University, Qufu, China. ${ }^{4}$ Department of Mathematics, Xiangtan University, Xiangtan, China. ${ }^{5}$ Faculty of Information Technology, Macau University of Science and Technology, Taipa, China.

\section{Publisher's Note}

Springer Nature remains neutral with regard to jurisdictional claims in published maps and institutional affiliations.

Received: 10 August 2019 Accepted: 20 January 2020 Published online: 03 February 2020

\section{References}

1. Laskin, N.: Fractional quantum mechanics. Phys. Rev. E 62(3), 3135 (2000)

2. Laskin, N.: Fractional quantum mechanics and Lévy path integrals. Phys. Lett. A 268(4-6), 298-305 (2000)

3. Naber, M.: Time fractional Schrödinger equation. J. Math. Phys. 45(8), 3339-3352 (2004)

4. Wang, S., Xu, M.: Generalized fractional Schrödinger equation with space-time fractional derivatives. J. Math. Phys. 48(4), $041502(2007)$

5. Guo, X., Xu, M.: Some physical applications of fractional Schrödinger equation. J. Math. Phys. 47(8), 082104 (2006)

6. Cheng, M.: Bound state for the fractional Schrödinger equation with unbounded potential. J. Math. Phys. 53(4), 043507 (2012)

7. Felmer, P., Tan, A.Q.J.: Positive solutions of the nonlinear Schrödinger equation with the fractional Laplacian. Proc. R. Soc. Edinb. 142(6), 1237-1262 (2012)

8. Wang, J.R., Zhou, Y., Wei, W.: Fractional Schrödinger equations with potential and optimal controls. Nonlinear Anal., Real World Appl. 13(6), 2755-2766 (2012)

9. Rida, S.Z., El-Sherbiny, H.M., Arafa, A.A.M.: On the solution of the fractional nonlinear Schrödinger equation. Phys. Lett. A 372(5), 553-558 (2008) 
10. Li, X., Xu, C.: A space-time spectral method for the time fractional diffusion equation. SIAM J. Numer. Anal. 47(3), 2108-2131 (2009)

11. Yildirim, A:: An algorithm for solving the fractional nonlinear Schrödinger equation by means of the homotopy perturbation method. Int. J. Nonlinear Sci. Numer. Simul. 10(4), 445-450 (2009)

12. Wei, L., He, Y., Zhang, X., Wang, S.: A numerical study based on an implicit fully discrete local discontinuous Galerkin method for the time-fractional coupled Schrödinger equation. Finite Elem. Anal. Des. 59, 28-34 (2012)

13. Wei, L., Zhang, X., Kumar, S., Yildirim, A.: A numerical study based on an implicit fully discrete local discontinuous Galerkin method for the time-fractional coupled Schrödinger system. Comput. Math. Appl. 64(8), 2603-2615 (2012)

14. Mohebbi, A., Abbaszadeh, M., Dehghan, M.: The use of a meshless technique based on collocation and radial basis functions for solving the time fractional nonlinear Schrödinger equation arising in quantum mechanics. Eng. Anal. Bound. Elem. 37(2), 475-485 (2013)

15. Baleanu, D., Inc, M., Aliyu, A.I., Yusuf, A.: Dark optical solitons and conservation laws to the resonance nonlinear Schrödinger's equation with Kerr law nonlinearity. Optik 147, 248-255 (2017)

16. Inc, M., Aliyu, A.I., Yusuf, A., Baleanu, D.: Dispersive optical solitons and modulation instability analysis of Schrödinger-Hirota equation with spatio-temporal dispersion and Kerr law nonlinearity. Superlattices Microstruct. 113, 319-327 (2018)

17. Inc, M., Yusuf, A., Aliyu, A.I., Baleanu, D.: Dark and singular optical solitons for the conformable space-time nonlinear Schrödinger equation with Kerr and power law nonlinearity. Optik 162, 65-75 (2018)

18. Garrappa, R., Moret, I., Popolizio, M.: Solving the time-fractional Schrödinger equation by Krylov projection methods. J. Comput. Phys. 293, 115-134 (2015)

19. Zhu, X., Yuan, Z., Wang, J., Nie, Y., Yang, Z.: Finite element method for time-space-fractional Schrödinger equation. Electron. J. Differ. Equ. 2017, 166, 1-18 (2017)

20. Wei, L., He, Y: Numerical algorithm based on an implicit fully discrete local discontinuous Galerkin method for the time-fractional KdV-Burgers-Kuramoto equation. Z. Angew. Math. Mech. 93(1), 14-28 (2013)

21. Kumar, S., Kocak, H., Yildirim, A.: A fractional model of gas dynamics equations and its analytical approximate solution using Laplace transform. Z. Naturforsch. A 67(6-7), 389-396 (2012)

22. Gupta, P.K., Singh, M., Yildirim, A.: Approximate analytical solution of the time-fractional Camassa-Holm, modified Camassa-Holm, and Degasperis-Procesi equations by homotopy perturbation method. Sci. Iran. 23(1), 155-165 (2016)

23. Yusuf, A., Inc, M., Aliyu, A.L., Baleanu, D.: Optical solitons possessing beta derivative of the Chen-Lee-Liu equation in optical fibers. Front. Phys. 7, 34 (2019)

24. Yusuf, A., Inc, M., Baleanu, D.: Optical solitons with M-truncated and beta derivatives in nonlinear optics. Front. Phys. 7, $126(2019)$

25. Kumar, D., Singh, J., Qurashi, M.A., Baleanu, D.: A new fractional SIRS-SI malaria disease model with application of vaccines, antimalarial drugs, and spraying. Adv. Differ. Equ. 2019(1), 278 (2019)

26. Oldham, K.B., Spanier, J.: The fractional calculus. Math. Gaz. 56(247), 396-400 (1974)

27. Lynch, V.E., Carreras, B.A., Del-Castillo-Negrete, D., Ferreira-Mejias, K.M., Hicks, H.R.: Numerical methods for the solution of partial differential equations of fractional order. J. Comput. Phys. 192(2), 406-421 (2003)

28. Langlands, T.A.M., Henry, B.I.: The accuracy and stability of an implicit solution method for the fractional diffusion equation. J. Comput. Phys. 205(2), 719-736 (2005)

29. Sun, Z., Wu, X.: A fully discrete difference scheme for a diffusion-wave system. Appl. Numer. Math. 56(2), 193-209 (2006)

30. Lin, Y., Xu, C.: Finite difference/spectral approximations for the time-fractional diffusion equation. J. Comput. Phys. 225(2), 1533-1552 (2007)

31. Grajales, J.C.M., Vargas, L.F.: Error analysis of a Fourier-Galerkin method applied to the Schrödinger equation. Appl. Anal. 95(1), 156-173 (2016)

32. Gong, Y., Wang, Q., Wang, Y., Cai, J.: A conservative Fourier pseudo-spectral method for the nonlinear Schrödinger equation. J. Comput. Phys. 328, 354-370 (2017)

33. Jagdev, S., Kumar, D., Baleanu, D.: On the analysis of fractional diabetes model with exponential law. Adv. Differ. Equ 2018(1), $231(2018)$

34. Amit, G., Singh, J., Kumar, D.: An efficient analytical approach for fractional equal width equations describing hydro-magnetic waves in cold plasma. Phys. A, Stat. Mech. Appl. 524, 563-575 (2019)

35. Jagdev, S., Kumar, D., Baleanu, D.: New aspects of fractional Biswas-Milovic model with Mittag-Leffler law. Math. Model. Nat. Phenom. 14(3), 303 (2019)

36. Singh, J., Kumar, D., Baleanu, D., Rathore, S.: On the local fractional wave equation in fractal strings. Math. Methods Appl. Sci. 42(5), 1588-1595 (2019)

37. Quarteroni, A.M., Valli, A.: Numerical Approximation of Partial Differential Equations. Springer, Berlin (1994) 